\title{
Los pronombres personales del mixteco: Un estudio com- parativo entre dos variantes lingüísticas
}

\section{Personal pronouns of mixtec: A comparative analysis be- tween two linguistic variant}

\author{
Ada R. Rázuri Martínez \\ Instituto Lingüístico de Verano \\ rosana_razuri@sil.org
}

\section{Resumen}

En este artículo se describe los pronombres personales de dos variantes lingüísticas del mixteco: San Bartolomé Yucuañe y Magdalena Peñasco, ambas localizadas en el distrito de Tlaxiaco, estado de Oaxaca, México. Las formas en las que ambas variantes lingüísticas clasifican los pronombres son divergentes. La primera codifica factores como el género, la edad y la cercanía del hablante y oyente. La segunda, en cambio, solo enfatiza el respeto hacia el oyente.

Palabras clave: Pronombres personales, variante lingüística, lenguas mixtecas

\begin{abstract}
This article describes and compares the personal pronouns of the Mixtec Language of the linguistic variant of San Bartolomé Yucuañe, with the linguistic variant of Magdalena Peñasco. Both are located in the district of Tlaxiaco, Oaxaca, Mexico. These are the ways the languages classify their pronoun changes from one to the other. The first, considers factors such as gender, age, and family and personal relationships. In the second variant of Mixtec, pronouns are only determined by the respect that is held with whom one is speaking. Considering these differences, we understand that these two variants of Mixtec have their unique way in using pronouns.
\end{abstract}

Keywords: Personal Pronouns, Linguistic Variant, Mixtec Languages 


\section{Introducción}

De las lenguas Oto-mangueanas proviene la familia mixtecana, que es una de las muchas lenguas que se hablan en México. Esta familia se divide en tres grupos lingüísticos que son como lenguas hermanas: el triqui, el cuicateco y el mixteco. Del cuicateco y el triqui han surgido pocas variantes lingüísticas, pero del mixteco, muchas. A los hablantes de este idioma se le llama "mixtecos", ellos no solamente se ubican en el sur del país, sino también en parte de Guerrero y Puebla.

Una de las particularidades que caracteriza a los mixtecos es su identidad de grupo, por la cual han surgido muchas variantes lingüísticas y siguen surgiendo en la actualidad. Muchas veces dos pueblos a penas pueden hablar exactamente de la misma manera; de hecho, en algunos casos existen diferencias notables incluso de un barrio a otro. Este fenómeno ha dado lugar a diferentes estimaciones del número de lenguas mixtecas. Ethnologue registra 52 variantes lingüísticas, mientras que el INALI (2010) registra 81.

Se dice que hay una división lingüística en el mundo mixteco entre los pueblos que usan /d/ y los que usan /s/ en las palabras como "oreja" y "sacerdote". En algunos pueblos estas palabras son do'o y dutu, y en otros son so'o y sutu. Los pueblos que usan $d$ se encuentran al noreste, y los que usan la $s$, al sureste.

Esta realidad nos motiva a investigar esas diferencias existentes entre un pueblo a otro; en este caso, el uso de los pronombres personales en dos variantes lingüísticas. En San Bartolomé Yucuañe (código ISO-mvg) hay cinco pronombres que indican primera persona (incluyendo al pronombre inclusivo plural); cuatro pronombres para segunda persona y cuatro pronombres para tercera persona (solamente con el rasgo [+humano]; en el grupo de pronombres de tercera persona (con el rasgo [-humano] hay siete pronombres; es decir, un pronombre para cada clasificación: animales, cosas esféricas, líquidos, deidades o personajes que se considera sagrados, objetos de madera o cosas manufacturadas, y las que caen en el rubro de pronombres que no pertenecen a las clasificaciones antes mencionadas.

Por lo antes dicho, lo que queremos lograr en este estudio es dar a conocer las diferencias de uso de los pronombres personales, según el contexto de uso de las dos variantes lingüísticas antes mencionadas. Se parte de un análisis base y luego se hace una comparación. Los datos adquiridos para el análisis base son de los nativohablantes de la comunidad de San Bartolomé Yucuañe, donde hace cuatro años vengo trabajando. Sin embargo, también se ha utilizado materiales 
extras como cuentos, cuestionarios, conversaciones, y por supuesto, la observación para confirmar algunos datos confusos. Para el estudio comparativo se ha utilizado la gramática de esta variante lingüística, que fue publicada en el 2013 por el Instituto Lingüístico de Verano, cuya autora, Barbara de Hollenbach, tuvo la amabilidad de proporcionarme datos extras y ayudarme a resolver algunas dudas.

\section{Categorías mentales que ordenan realidades}

Desde el punto de vista de la gramática tradicional, «el pronombre es una de las diez partes de la oración, la cual se declina por casos» (Nebrija, 1984 [1492], p. 180). Del mismo modo se dice que «con la denominación de pronombres personales se agrupan varias palabras, en número limitado, cuyo contenido se refiere a la noción de persona gramatical. Esta noción abarca a los tres elementos externos a la lengua que intervienen en todo acto de habla: el hablante, el interlocutor y el conjunto de todo lo demás» (Alarcos, 1994, p. 70). Pero si vamos un poco más a fondo observaremos que "los pronombres tienen que ver con las interrelaciones de las personas, esto es, de entes reales, como componentes del acto comunicativo básico, o sea, «hablante/oyente/cosa mentada», pero ello constituye solo un punto de partida” (Gallardo, 2004, p. 98). Esto quiere decir que «al utilizar el lenguaje contribuimos a crear la realidad que intentamos representar» (Duranti, 2000, p. 291). Sin embargo, no nos podemos quedar con este concepto superficial porque no llegaremos a comprender el modo de cómo el lenguaje va fusionado con la cultura, y más aún, de qué manera los hablantes de un idioma clasifican los símbolos/signos en su mente de tal modo que lo que dicen es meramente un conocimiento preconcebido y natural dentro de su cultura, porque para esto, debemos saber que «los símbolos son categorías mentales que los humanos crean y rotulan para comprender y ordenar las realidades en las que viven (...), y los símbolos juegan un papel clave para que las personas comprendan el mundo; dado que, organizan las experiencias en categorías mentales. Para comprender el mundo los individuos deben reducirlo a un número manejable de categorías» (Hiebert y otros, 2009, p. 269).

\section{Los pronombres personales en contexto}

Zilstra (1995) afirma que el sistema pronominal es una clave importante para entender el mundo mixteco. Ellos tienen diversos pronombres para organizar sus conocimientos, por lo que ella establece la siguiente clasificación: "pronombres para los seres humanos masculinos, los seres 
humanos femeninos, los animales, los espíritus y lo que se considera divinidad, tales como el dios de la lluvia, las estrellas, la luna, el sol y hasta el sacerdote; también el líquido, la madera, las cosas esféricas y los objetos inanimados tienen pronombres propios...» (Zilstra, 1995, p. 6).

Esta es una visión general de la clasificación pronominal en el mixteco porque cabe mencionar que cada variante lingüística tiene sus propias particularidades. Digo esto porque en San Bartolomé Yucuañe hay factores específicos que influyen en esta clasificación. En el apartado siguiente los mencionaré.

Otra característica importante en la cultura mixteca es que «tener un pronombre separado para los líquidos es una expresión no solamente de la importancia tradicional del dios Savi de la lluvia, de quien los mixtecos derivan el nombre para sí mismos: La gente de la Lluvia. El dios de la lluvia es una de muchas deidades y espíritus, pero considerando su importancia, no se refieren a él con el pronombre "regular" para la divinidad, sino con un pronombre especial, que entonces, probablemente por asociación, se confiere al resto de líquidos» (Zilstra, 1995, p. 6). Por eso, estoy de acuerdo cuando Benveniste (1971) afirma que «los pronombres personales son realidades de discurso, y así el lenguaje reproduce el mundo sometiéndolo a su propia organización o cosmovisión» (p. 27).

\section{Factores que influyen en la clasificación de los pronombres personales}

Los tratamientos de cortesía es un factor importante para considerar cuando se trata de analizar los pronombres personales, sabiendo que cada cultura maneja un conjunto de parámetro diferente. En el caso de la variante lingüística de SBY, se observa que la familiaridad, la edad, el respeto y la cercanía entre hablante, oyente y referente son factores que influyen en la clasificación. Por lo que la definición de Criado de Val (1972) es acertado en cuanto a los tratamientos, pues él afirma que esto se fundan en la estimación mutua de los interlocutores. Además, afirma que es preciso, para su descripción, la relación previa entre estos y su contexto mental.

Criado de Val (1972) también afirma que:

En todo diálogo hay una mutua valoración (categoría social, grado de familiaridad, edad, etc.) de los interlocutores. La expresión más directa de esta valoración por el lenguaje son los tratamientos, cuyo sistema 
varía grandemente de unos idiomas a otros. Estoy de acuerdo con él cuando afirma que algunos idiomas a penas pueden diferenciar un tratamiento de cortesía con otro; sin embargo, hay otros en el que es necesario el conocimiento no solo del idioma, sino de las costumbres sociales para acertar con el tratamiento oportuno y justo.

(Criado de Val, 1972, p. 179)

Del mismo modo, otro factor importante es el género, pues también influye en la clasificación de los pronombres. Digo esto porque, mientras recogía los datos, pude darme cuenta que muchos de los pronombres no encajaban solamente considerando el uso de los tratamientos de cortesía. La realidad de la mixteca nos enseña que en una sociedad jerarquizada también se puede hacer distinción de género y no solamente de nivel social. Duranti (2000) dice que ciertas expresiones parecen estar asociadas a hablantes varones o mujeres, o porque hay una actitud o una postura afectiva que se asocia con un género y no con el otro. En la actualidad, aunque no en todas las variantes lingüísticas, se observa este hecho.

Finalmente, es importante recalcar que cada cultura tiene la capacidad de clasificar según su contexto. Para este caso, existe un grupo de pronombres personales que clasifica a un grupo con rasgo [-humano], a lo que yo le llamo "Los otros pronombres". Se dice que el lenguaje es un signo; por tanto, debemos tomar en cuenta la manera en que estos signos hacen que los seres humanos formulen estructuras simbólicas, establezcan clasificaciones, jerarquías, etc. Hiebert y otros (2009) sostiene: «un signo es algo que representa (simboliza, significa, evoca, recuerda, señala, encarna, tipifica, denota se refiere a) alguna otra cosa en la mente de una persona o una comunidad» (p.266). Esta definición nos muestra que los pronombres son un vehículo que expresa la realidad de una comunidad en particular, sabiendo que un símbolo es un tipo de signo. Entiéndase símbolo como: «categorías mentales que los humanos crean y rotulan para comprender y ordenar las realidades en las que viven» (Hiebert, 2009, p. 268). En este sentido, entendemos que este es el recurso que una comunidad toma para crear, pensar, rotular y expresar su conocimiento del mundo.

\section{Descripción de los pronombres personales de San Bartolomé Yucuañe ${ }^{1}$}

Primero cabe decir que los pronombres personales en el mixteco se dividen en dos campos: dependientes e independientes (en este grupo se considera también a los pronombres compuestos con maa "enfático"). La 
clasificación de los pronombres personales dependientes de esta variante lingüística está influenciada por el género, edad, respeto, familiaridad y cercanía. En el apartado 4.2 se muestra un cuadro de estos pronombres, según su contexto de uso. Pero a grandes rasgos menciono que hay cinco pronombres para primera persona (incluyendo al pronombre inclusivo plural); cuatro pronombres para segunda persona; cuatro pronombres para tercera persona (solamente con el rasgo [+humano]. El grupo de pronombres de tercera persona (con el rasgo [-humano] se muestra en otro cuadro, estas hacen referencia a animales, cosas esféricas, líquidos, deidades o personajes que se considera sagrados y un pronombre para objetos de madera o cosas manufacturadas que caen en el rubro de "Los pronombres de otros géneros".

\subsection{Pronombres independientes}

Los pronombres independientes son los que pueden funcionar por sí mismos dentro o fuera de una oración. Estos pronombres generalmente se usan en un discurso. No estudiaremos este tema aquí, pero se hará mención de algunos ejemplos.

(1) Maa sa nani Carmen.

ENF 1R IMPFV \llamarse Carmen

'Yo me llamo Carmen'.

(2) Saña ka'an.

1R IMPFV \hablar

'Yo hablo'.

$$
\begin{aligned}
& \text { Ndijin, jini ni ka'vi ni. } \\
& \text { 2R IMPFV } \backslash \text { saber } 2 \mathrm{R} \text { IMPFV } \backslash \text { leer } 2 \mathrm{R} \\
& \text { 'Usted sabe leer'. }
\end{aligned}
$$

\subsection{Pronombres dependientes}

Los pronombres dependientes básicamente se clasifican de la siguiente manera: respeto, familiar y cercanía. Dentro de ella se involucran los factores que mencionamos anteriormente: género y edad. Estos pronombres son formas enclíticas y se apoyan en la palabra anterior. Además no tienen un significado propio cuando están aislados de la oración. 
Los pronombres dependientes en el mixteco hacen referencia al hablante, al oyente o a un referente (tercera persona). Estas expresan el sujeto del verbo, el complemento de una preposición, el poseedor de un sustantivo y también pueden expresar el complemento del verbo. Así mismo, se presentan solamente cuando no hay un sustantivo o frase nominal que exprese el sujeto, complemento o poseedor.

En el siguiente cuadro se muestra el panorama general de la clasificación de los pronombres con el rasgo [+ humano]. Después de este cuadro se muestra algunos ejemplos de cada uno de ellos y una breve descripción de acuerdo a su contexto de uso.

\begin{tabular}{|l|l|l|l|l|}
\hline Pronombre & Persona & $\begin{array}{l}\text { Género del ha- } \\
\text { blante }\end{array}$ & $\begin{array}{c}\text { Género del } \\
\text { Oyente - Refe- } \\
\text { rente }\end{array}$ & Contexto \\
\hline sa & $1 \mathrm{R}$ & Varón-Mujer & Varón-Mujer & Respeto \\
\hline li & $1 \mathrm{FM}$ & Mujer & Varón-Mujer & Familiaridad \\
\hline ndi & $1 \mathrm{FV}$ & Varón & Varón & Familiaridad \\
\hline ta & $1 \mathrm{INV}$ & Varón & Varón-Mujer & Intimidad \\
\hline o & $1 \mathrm{INCL}$ & Varón-Mujer & Varón-Mujer & $\begin{array}{l}\text { Incluye al } \\
\text { oyente }\end{array}$ \\
\hline ni & $2 \mathrm{R}$ & Varón-Mujer & Varón-Mujer & Respeto \\
\hline lo & $2 \mathrm{FM}$ & Mujer & Varón-Mujer & Familiaridad \\
\hline ndo & $2 \mathrm{FV}$ & Varón & Varón-Mujer & Familiaridad \\
\hline ro & $2 \mathrm{FA}$ & Anciana & Varón-Mujer & Familiaridad \\
\hline rre & $3 \mathrm{RM}$ & Mujer & Varón & Respeto \\
\hline de & $3 \mathrm{RV}$ & Varón & Varón & Respeto \\
\hline ña & $3 \mathrm{M}$ & Varón-Mujer & Mujer & Respeto \\
\hline i & $3 \mathrm{~F}$ & Varón-Mujer & $\begin{array}{l}\text { Varón-Mu- } \\
\text { jer-niño-gente } \\
\text { común }\end{array}$ & Familiaridad \\
\hline
\end{tabular}

Cuadro 1. Pronombres según el contexto 


\subsubsection{Los pronombres dependientes de respeto}

El pronombre de respeto u honorífico no cambia según el género. Así que $s a$ equivale a yo cuando se considera al oyente como alguien a quien se debe respeto y ni equivale a usted. Pero no sucede lo mismo con los de tercera persona, porque cuando el oyente es de género masculino el hablante varón usa de (él), y las mujeres usan rre (él). Sin embargo, cuando el oyente es de género femenino el pronombre es el mismo; es decir, tanto el hablante masculino y femenino usan el pronombre $\tilde{n} a$ (ella).
Tetatna, tnau'u
kini
sa.
doctor IMPFV $\backslash$ doler cabeza $1 \mathrm{R}$
'Doctor, me duele la cabeza'.

(5)

$\begin{array}{lllcll}\text { Vaji } & \text { sa } & \text { vekikaxtnu'u } & \text { sa } & \text { ja } & \text { nasa'a } \\ \text { IMPFV:venir } & \text { 1R } & \text { INC:IRR\PL:decir } & \text { 1R } & \text { para } & \text { REP:hacer } \\ \text { 'Vine para decirle } & & & & \end{array}$

nuu ni iin jandiuxi ni ja ka'an nuu ñayivi yu. a $2 \mathrm{R}$ un favor $2 \mathrm{R}$ para IRR/hablar a persona ADL a usted que me haga el favor de hablar con esa persona'.

(6) $\mathrm{Na}$ orre ko de yutnee.

qué hora IRR:estar 3RV mañana '¿Qué hora estará mañana?'

(7) Vaji

sa vekikoto

sa tekunija'nu yu. ¿Nu iyo rre? IMPFV $\backslash$ venir 1R INC:IRR\PL:ver 1R presidente ADL si IMPFVlestar 3RM 'Vine para hablar con el presidente. ¿Está él?'

(8) Te maa ña chi ñatuu nitna ini ña ja ki'in ña yuku. y ENF 3M pues NEG PFV:gustar 3M para IRR $\backslash$ ir $3 \mathrm{M}$ cerro 'A ella no le gustaba ir al campo'. 


\subsubsection{Pronombres dependientes de familiaridad}

Para expresar familiaridad se usan pronombres como ndi, para primera persona, cuando el hablante es varón; cuando el hablante es mujer se usa li. Para segunda persona, se usan pronombres como ndo cuando el hablante es varón, y cuando el hablante es mujer se usa lo; también se usa ro, cuando el hablante es una mujer anciana. Para tercera persona, en este contexto de familiaridad, se usa el pronombre $i$ (él/ella), cuando el referente es un niño o alguien de menos edad que el hablante, o a veces una persona desconocida. Es más o menos como "tutear" en español.

(9) Te ki'in ndi ji'in se'e ndi ya'a.

y IRR \ir 1FV con hijo 1FV este

'Me voy con mi hijo'.

(10) ¿Skee-ka li jaku ndeyu ya'a?

IMPFV\servir-más 1FM poco comida este

'¿Te sirvo más comida?'

(11) Saka'nu ini ndo, vaa Juaa.

IMPFV:disculpar 2FV compadre Juan.

'Disculpeme, compadre Juan'.

(12) Nawa sa'a lo.

qué IMPFV $\backslash$ hacer $2 \mathrm{FM}$

¿Qué haces?'

(13) Te kotondee ro chi sanaani-ka chi kixi rre.

y IRR \mirar 2FA pues de repente-más pues IRR\dormir 3RM

'Te fijas, porque no vaya ser que se duerma'.

(14) Manuel ja nikukaji ini naxa satniñu i.

Manuel ya PEFV:aprender cómo IMPFV \trabajar 3F

'Manuel ya sabe cómo trabajar'.

\subsubsection{Pronombre dependiente de cercanía}

Hay un tercer contexto, se trata del pronombre de primera persona ta que expresa amistad cercana con el oyente: amigos, esposos, padre e hijos (as). 


$\begin{array}{llll}\text { Masu yii ndo } & \text { kuu } & \text { ta. } \\ \text { NEG esposo } & \text { 2FV } & \text { PRS:ser } & \text { IINV } \\ \text { 'No soy tu esposo'. } & & \end{array}$

4.2.4 Los pronombres de otros géneros

Existe otro grupo de pronombres personales de tercera persona con rasgo [-humano]. Cumplen la misma función de pronombre personal y se presenta en el mismo orden sintáctico dentro de la oración que el resto de pronombres.

\begin{tabular}{|c|c|c|}
\hline Pronombre & Persona & Referente \\
\hline $\mathrm{ti}$ & $3 \mathrm{~A}$ & Referente a animales \\
\hline $\mathrm{ti}$ & $3 \mathrm{E}$ & Referente a objetos esféricos \\
\hline $\mathrm{rre} / \mathrm{de}$ & $3 \mathrm{~L}$ & Referente a líquido \\
\hline $\mathrm{ya}$ & $3 \mathrm{D}$ & Deidad o lo que merece reverencia \\
\hline $\mathrm{nu}$ & $3 \mathrm{MD}$ & $\begin{array}{c}\text { Objetos de madera y demás objetos } \\
\text { manufacturados }\end{array}$ \\
\hline $\mathrm{i}$ & $3 \mathrm{C}$ & $\begin{array}{c}\text { Para cosas que no tienen pronombre } \\
\text { propio }\end{array}$ \\
\hline
\end{tabular}

\section{Cuadro 2. Pronombres de otros géneros}

El pronombre ti se utiliza para hacer referencia a los animales.
(16) Iin chuun nitnii ti in tniñi ji'in tniñi ti.
un tecolote PFV:agarrar $3 \mathrm{~A}$ un ratón con uñas $3 \mathrm{~A}$
'Un tecolote agarró un ratón con sus uñas'.

El otro pronombre que se escribe igual que el pronombre para animal, ti. Se usa para todo lo que culturalmente se considera un objeto esférico (palta, tomate, naranja, ajo, etc.).

Kuii ini ti te kuii yika ti
verde dentro $3 \mathrm{E}$ y verde espalda
'Verde por dentro y verde por fuera,


te iyo yiki ti ko masu ñayivi kuu ti

y IMPFV:tener hueso $3 \mathrm{E}$ pero NEG persona IMPFV $\backslash$ ser $3 \mathrm{E}$ tiene hueso, pero no es persona'.

El siguiente pronombre es para referirse a los líquidos, rre. Esta clasificación es importante; dado que el agua determina las cosechas necesarias para la supervivencia.

Ja nichaa rre.

ya PFV:regresar 3L

'Ya regresó el agua'.

El pronombre de deidad ya hace referencia a lo que culturalmente se considera seres sagrados, como el sol, la luna, la lluvia y las estrellas. Hay una importancia tradicional del dios Savi de la lluvia, de quien los mixtecos derivan el nombre para sí mismos: La gente de la lluvia (Zylstra 1995, p. 4).

(19) Nika'an iin yaa choxiñi nuu sa te nika'an sa ji'in ya. PFV:hablar un dios estrella a 1R y PFV:hablar 1R con 3D 'Me habló la estrella. Yo hablé con ella'.

(20) I'ni xeen.

caliente mucho

'¡Qué calor!'

—Jon, xeen ka'yu sa'a ya.

sí, mucho IMPFV \quemar IMPFV $\backslash$ hacer 3D

'Sí, hace quemar mucho el sol'.

También en este grupo se incluye al sacerdote como una deidad, quizá porque cumple un rol religioso, que para ellos es muy respetado.

(21) Kee santu yu ichi te no'o ya veñu'u.

IMPFV:venir santo ADL camino y IRR $\backslash$ ir 3D iglesia

'Viene el santo por aquí, luego se va a la iglesia'. 
Los pronombres personales del mixteco: Un estudio comparativo entre dos variantes lingüísticas

Antiguamente, las enfermedades eran consideradas deidades que hacían mal a la gente.

(22) Nda'vi xeen sa'a ña ya. Chi'i xeen ña ka'ni. pobre mucho IMPFV $\backslash$ hacer CC 3D IMPFV \agarrar mucho CC calentura

'Pobrecita, está muy enferma. Tiene fiebre (lit. a causa del tiempo)'.

El pronombre $n u$ se usa para hacer referencia a árboles, madera y objetos manufacturados.

(23) Leslie nija'nu i nduku yu te Pelu nijiso i nu. Leslie PFV:cortar 3F leña ADL y Pedro PFV:cargar 3F 3MD 'Leslie cortó la leña y Pedro lo cargó'.
Nuja nakoo camión ja ne'e xeen yukuan.

Ojalá REP:haber carro ya IMPFV\amanecer muy ahí 'Ojalá haya camión temprano'.

-Jon, weno jon koo nu.

Sí seguro sí IRR\haber 3MD

'Sí, seguramente habrá'.

Finalmente, el pronombre $i$ se usa para asignar cosas que no tienen su propio pronombre; también algunas veces se usa para algo abstracto como los espíritus y ciertas enfermedades.
Sa'a
dañu
tna'a
tinuu
o.
IMPF\hacer daño $3 \mathrm{~N}$ también ojos
'(La presión alta) también daña los ojos'.

(26) Maa li chi ñatuu iyo li tachi ko iku

ENF $1 \mathrm{~F}$ pues NEG IMPFV \hay $1 \mathrm{~F}$ diablo pero ayer

chi nika-siu'u ña i.

pues PFV:PL:asustar CC $3 \mathrm{~N}$

'No le tengo miedo a los espíritus, pero ayer me asustaron'. 


\section{Análisis comparativo: San Bartolomé Yucuañe y Magdalena Peñasco}

Lo que se puede observar es que en la variante de Magdalena Peñasco la clasificación pronominal se divide en dos: respeto y familiaridad, sin considerar el género como en la de San Bartolomé Yucuañe. Por otro lado, lo que ambas variantes lingüísticas sí comparten sin diferencia son los pronombres que indican respeto, tanto para primera y segunda persona. Pero en tercera persona la situación cambia.

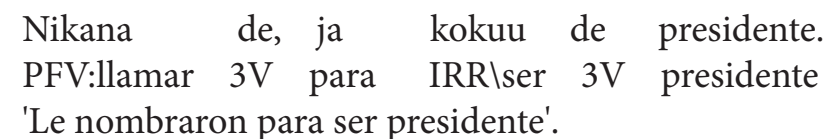

$$
\begin{aligned}
& \text { Na'a jana'a ma nikayokaa } \quad \text { ña ñ'i. } \\
& \text { Mujer antes DEF PFV:PL:HBT:subir } 3 \mathrm{M} \text { temascal } \\
& \text { 'Las mujeres de antaño acostumbraban bañarse en el temascal2'. }
\end{aligned}
$$

En la variante de SBY influye el género del hablante y oyente. Es decir, cuando el hablante y el oyente es varón, se usa el pronombre de (él); cuando el hablante es mujer y el oyente es varón, se usa el pronombre rre (él). Finalmente, cuando el hablante es varón/mujer y el oyente es mujer, se usa el pronombre ña (ella).

También hay diferencias cuando nos referimos a los pronombres familiares, porque en la variante de MgP, se usan los pronombres ri "yo"; ro "tú"; $i$ "él/ ella". "Aunque hoy en día el uso de ri y ro está extendiéndose entre adultos; esto probablemente refleja la extensión de tú en español durante las últimas décadas. Sin embargo, algunos en la Sección Primera de Magdalena no usan ro ni ri; hablan siempre de ni y sa con sus hijos y con sus esposos; es decir, no hacen diferencia entre formal y familiar. Aún entre niños usan ni y sa» (Erickson, 2013, p. 48)

(29) Kuan koo ri nuya'vi.

INClir PLIIRR 1F plaza

'Vamos a la plaza'.

(30) Chinee tutu ro a nuu bolsa ro a te ki'in ro skuela. IRR:meter papel 2F DFC cara bolsa 2F DFC y IRR Iir 2F escuela 'Mete tus papeles en tu bolsa y vete a la escuela'. 
En SBY el factor género vuelve aparecer. Tenemos ndi (yo-hablante varón); li (yo-hablante mujer); ndo (tú-hablante varón); lo (tú-hablante mujer); ro (tú-hablante mujer anciana). Y por si fuera poco también existe en esta variante un pronombre que indica cercanía o intimidad, ta (yo-hablante varón). El siguiente cuadro muestra las diferencias en ambas variantes lingüísticas.

\begin{tabular}{|c|c|c|c|}
\hline & $\begin{array}{l}\text { Mixteco de } \\
\text { SBY }\end{array}$ & $\begin{array}{l}\text { Mixteco de } \\
\text { MgP }\end{array}$ & Español \\
\hline \multicolumn{4}{|l|}{ Primera persona } \\
\hline Familiar mujer (AG) & li & ri & \multirow{4}{*}{ yo } \\
\hline Respeto AG (AG) & sa & sa & \\
\hline Familiar varón (AG) & ndi & ri & \\
\hline $\begin{array}{l}\text { Familiar íntimo } \\
\text { varón }(A G)\end{array}$ & ta & - & \\
\hline Plural inclusivo AG (AG) & $\mathrm{o}$ & $\mathrm{o}$ & nosotros \\
\hline \multicolumn{4}{|l|}{ Segunda persona } \\
\hline Respeto AG (AG) & ni & $\mathrm{ni}$ & usted \\
\hline Familiar mujer (AG) & lo & \multirow[t]{3}{*}{ ro } & \multirow[t]{3}{*}{ tú } \\
\hline Familiar varón (AG) & ndo & & \\
\hline Familiar anciana (AG) & ro & & \\
\hline \multicolumn{4}{|l|}{ Tercera persona } \\
\hline Respeto mujer (V) & rre & de & \multirow[t]{4}{*}{ él/ella } \\
\hline Respeto varón (V) & de & de & \\
\hline Respeto AG (M) & ña & ña & \\
\hline Familiar AG (AG) & $\mathrm{i}$ & $\mathrm{i}$ & \\
\hline Seres sagrados o deidad & ya & ya & \multirow{5}{*}{ - } \\
\hline Líquido & $\mathrm{de} / \mathrm{rre}$ & de & \\
\hline Objetos esféricos & $\mathrm{ti}$ & $\mathrm{ti}$ & \\
\hline Animales & ti & $\mathrm{ti}$ & \\
\hline $\begin{array}{l}\text { Madera u objetos manufac- } \\
\text { turados }\end{array}$ & $\mathrm{nu}$ & tnu & \\
\hline
\end{tabular}




\section{Buscando familiaridad en los pronombres personales}

Dado que el español es un idioma familiar para muchos, hacer una comparación final entre el español y el mixteco ayudará a ver las diferencias entre una y la otra.

(a) En el español la marcación de persona es parte de la conjugación del verbo, pero en el mixteco no.

(b) En el español los pronombres son usados optativamente para dar énfasis, pero en el mixteco generalmente son obligatorios.

(c) En español hay casos para el sujeto, complemento directo, complemento indirecto, término de preposición y poseedor, en cambio el mixteco no tiene casos.

(d) En español la forma plural no diferencia entre inclusivo y exclusivo como lo hace el mixteco. En el mixteco la forma inclusiva o nosotros es diferente a la forma exclusiva sa; En el primer caso, se emplea para un grupo que incluye a los oyentes. Cuando se usa para varias personas, generalmente, se usa una partícula que señala el plural. También se usa cuando una persona está pensando o hablándose así mismo. Por otro lado, la forma exclusiva se expresa con sa cuando no incluye (si es uno o más de uno) al oyente o los oyentes.

(e) En español hay diferentes pronombres para singular y plural, en cambio en el mixteco se usan los mismos pronombres para expresar esto.

(f) En el español hay pronombres para respeto y familiar solamente en segunda persona; sin embargo, en el mixteco de SBY y MgP hay pronombres de respeto y familiares para primera, segunda y tercera persona. En el mixteco de SBY estos pronombres familiares incluyen factores como edad, género. También esta variante hay un pronombre que expresa otro grado de intimidad.

(g) En el español hay dos géneros gramaticales, en el mixteco hay dos géneros naturales para personas adultas, más otro género para jóvenes y niños, para animales, líquido, cosas esféricos, seres sagrados y objetos de madera o manufacturados. Estos géneros son parcialmente naturales y parcialmente culturales.

(h) En el español no hay diferencias para marcar el género del hablante u oyente, pero en el mixteco de SBY se dan estas diferencias. 
Los pronombres personales del mixteco: Un estudio comparativo entre dos variantes lingüísticas

(i) El español carece de pronombre para el complemento conocido; sin embargo, el mixteco tiene un pronombre para indicar complementos sin especificar la persona.

\section{Conclusiones}

1. "Las culturas son los mundos mentales que las sociedades construyen para vivir en un mundo confuso: para darle orden y significado; y los símbolos son ladrillos que se usan para construir estos mundos" (Paul G. Hiebert y otros, 2009 , p. 269). Del mismo modo, el autor afirma que "los símbolos son categorías mentales que los humanos crean y rotulan para comprender y ordenar las realidades en las que viven. Dado que, sin ellos los individuos no pueden pensar y ordenar las realidades en las que viven". También Becker (2002) afirma que "La lengua es un sistema de medios de expresión apropiados para un fin. [Por tal motivo] "al analizar cualquier hecho lingüístico hay que tomar en cuenta su función que cumple en el proceso social. Esta función es como un conjunto de estrategias simbólicas que forman parte del tejido social y de la representación individual de mundos posibles y reales". (Duranti 2000, p. 22). En la lengua mixteca los pronombres expresan esta realidad; es por ello que a través de ella, podemos conocer la manera en que esta cultura piensa. Una evidencia son los factores involucrados en el momento de clasificar. Por eso ahora tenemos claro que el sistema de pronombres mixtecos es una clave importante en la cultura.

2. Sin embargo, cabe mencionar que no todas las culturas usan los mismos criterios para clasificar los pronombres. Como hemos visto, ni siquiera es lo mismo en las variantes del mismo idioma, los resultados de este estudio comparativo hace evidente este hecho. Entonces, podemos afirmar que hay variantes lingüísticas del mixteco donde factores como el género, la edad, la familiaridad e intimidad es importante, y en otras solamente el factor respeto y familiaridad son considerados. 


\section{Referencias bibliográficas}

Alarcos, E. (1994). Gramática de la Lengua Española. Primera edición. Madrid: Espasa-Calpe.

Benveniste, É. (1971). La naturaleza de los pronombres. En Problemas de Lingüística General. México: Siglo XXI.

Becker, A. (2002). Análisis de la Estructura pragmática de la cláusula en el español de Mérida (Venezuela). Recuperado de http://elies.rediris.es/elies17/index. htm

Criado de Val, M. (1972). Fisionomía del español y de las lenguas modernas. Madrid: SAETA.

De Nebrija, A. 1984 [1492]. Gramática de la lengua castellana. Estudio y edición de Antonio Quilis. Madrid: Editora Nacional. Recuperado de http://www.filos. unam.mx/LICENCIATURA/Pagina_FyF_2004/introduccion/Gramatica_Nebrija.pdf

Duranti, A. (2000). Antropología Lingüística. Primera edición en Cambridge University Press. Madrid, España: Lavel, S.A.

Erickson de Hollenbach, E. (2013). Gramática del mixteco de Magdalena Peñasco (Sa'an ñuu savi). Primera edición. Serie de gramáticas de lenguas indígenas de México 13. E.U.A.: En los talleres de Snowfall Press, Phoenix, AZ.

Paul G. Hiebert, R. Daniel Shaw, Tite Tienou. (2009). Hacia un entendimiento de las religiones populares: una respuesta cristiana a las creencias y las prácticas populares. Primera edición. Córdoba: Recursos Estratégicos Globales (REG).

Instituto Nacional de Lenguas Indígenas. (2010). Catálogo de las lenguas indígenas nacionales. Agrupación lingüística: mixteco. Familia lingüística: Oto-mangue. México. Recuperado de http://www.inali.gob.mx/clin-inali/html/1_mixteco.html

Instituto Lingüístico de Verano (ILV, A.C.). (2016). La familia mixteca. Oaxaca. Recuperado de http://www-01.sil.org/mexico/mixteca/00e-mixteca.htm 
Zylstra, C. F. (1995). Cosmovisión Mixteca. Material de enseñanza. E.U.A.: Seminario Fuller, clase dirigida por el Dr. Charles Kraft.

${ }^{1}$ Abreviaturas utilizadas: $1 \mathrm{~F}=$ primera persona familiar; $2 \mathrm{~F}=$ segunda persona familiar; $3 \mathrm{M}=$ tercera persona mujer; $3 \mathrm{~V}=$ tercera persona varón; $\mathrm{ADL}=$ artículo definido lejano; $\mathrm{AG}=$ ambos géneros; CC: complemento conocido; $\mathrm{DEF}=$ artículo definido; $\mathrm{DFC}=$ artículo definido cercano; IRR = irrealis; HBT = habitual; IMP = imperativo ; INC = incoactivo; MgP = Magdalena Peñasco; $\mathrm{NEG}=$ negación: $\mathrm{PL}=$ plural; $\mathrm{PFV}=$ perfectivo; $\mathrm{IMPFV}=$ imperfectivo; $\mathrm{REP}=$ repetitivo; $\mathrm{SBY}=$ San Bartolomé Yucuañe; $\mathrm{V}=$ varón; $1 \mathrm{R}$ = primera persona respeto; $1 \mathrm{FM}=$ primera persona familiar (hablante mujer); $1 \mathrm{FV}=$ primera persona familiar (hablante varón); $1 \mathrm{INCL}=$ primera persona inclusivo; $1 \mathrm{INV}=$ primera persona-cercano (hablante varón); $2 \mathrm{R}=$ segunda persona respeto; $2 \mathrm{FM}$ = segunda persona familiar (hablante mujer); $2 \mathrm{FV}$ = segunda persona familiar (hablante varón); 2FA = segunda persona familiar (anciana); $3 \mathrm{RM}=$ tercera persona respeto (hablante mujer); $3 \mathrm{RV}$ = tercera persona respeto (hablante varón); $3 \mathrm{M}=$ tercera persona respeto femenino (hablante ambos géneros); $3 \mathrm{~N}=$ tercera-neutro; $3 \mathrm{~A}=$ animales; $3 \mathrm{E}=$ objetos esféricos; $3 \mathrm{~L}=$ líquidos; $3 \mathrm{D}=$ deidad; $3 \mathrm{MD}=$ objetos de madera o manufacturados.

${ }^{2}$ Recinto tipo horno artesanal que alberga a más de una persona. Se usa para hacer baños medicinales a vapor o para realizar rituales. 